\title{
DISPLAYS: Trends and Technology I PROJECTION: Technologies, Systems and Applications
}

\author{
Moderador: Claudio Younis \\ Chief Executive Officer (CEO) at ELETRO EQUIP / Director at \\ SET \\ Speaker: Daniel Monteiro Pereira \\ Gerente de vendas / Christie Digital Systems \\ Speaker: Gabriel Gonçalves \\ Regional Product Manager / Epson America Inc. \\ Speaker: Rodrigo Leme \\ Service Engineer / SONY \\ Speaker: Clayton Brito \\ General Manager / Christie Digital Systems \\ Speaker: Alexandre Fontes \\ Supervisor Pré-Vendas / LG Electronics \\ Speaker:: Milton Souza
}

Pre Sales Manager, B2B da empresa Samsung Eletronics 


\section{Consumption \& Audio Visual Production}

\section{DISPLAYS: Trends and Technology I PROJECTION: Technologies, Systems and Applications}

\section{Moderator: Claudio Younis}

Chief Executive Officer (CEO) at ELETRO EQUIP / Director at SET

This Panel will demonstrate and discuss the different technologies available in professional displays and their commercial applications. The points covered in the presentation will be:

- The benefits of professional monitors in relation to televisions sets for consumers;

- LCD, LED, OLED;

- Overhead projection cubes and micro cubes;

- LED panels;

- Video walls.

\section{Speaker: DANIEL MONTEIRO PEREIRA}

Gerente de vendas / Christie Digital Systems

Speaker: GABRIEL GONÇALVES

Regional Product Manager / Epson America Inc.

Speaker:RODRIGO LEME

Service Engineer / SONY

Speaker:CLAYTON BRITO

General Manager / Christie Digital Systems

Speaker: ALEXANDRE FONTES

Supervisor Pré-Vendas / LG Electronics

Speaker:MILTON SOUZA

Pre Sales Manager, B2B da empresa Samsung Eletronics 


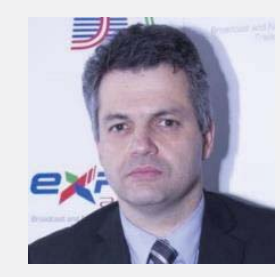

\section{CLAUDIO YOUNIS}

Chief Executive Officer (CEO) at ELETRO EQUIP / Director at SET

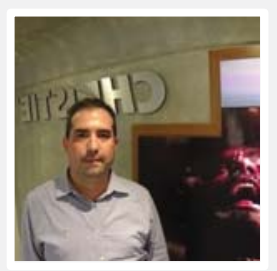

\section{DANIEL MONTEIRO PEREIRA}

Gerente de vendas / Christie Digital Systems

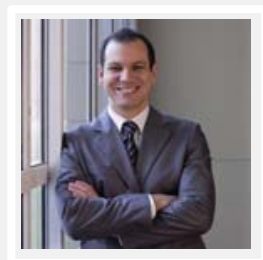

\section{GABRIEL GONÇALVES}

Regional Product Manager / Epson America Inc.

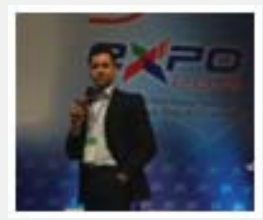

\section{CLAYTON BRITO}

General Manager / Christie Digital Systems

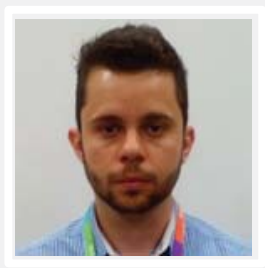

\section{RODRIGO LEME \\ Service Engineer / SONY}

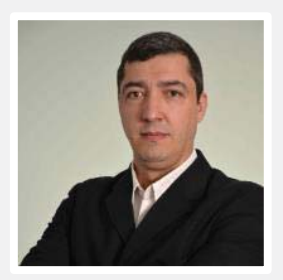

\section{ALEXANDRE FONTES}

Supervisor Pré-Vendas / LG Electronics

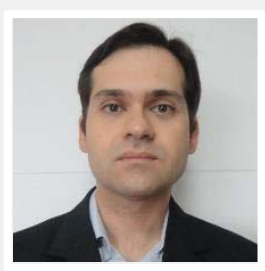

\section{MILTON SOUZA}

Pre Sales Manager, B2B da empresa Samsung Eletronics 